PHYSICAL REVIEW D 94, 089902(E) (2016)

\title{
Erratum: Improving cosmic string network simulations [Phys. Rev. D 90, 043539 (2014)]
}

Mark Hindmarsh, Kari Rummukainen, Tuomas V. I. Tenkanen, and David J. Weir

(Received 12 October 2016; published 26 October 2016)

DOI: 10.1103/PhysRevD.94.089902

In our paper, we stated that the algorithm for the numerical solution of the partial differential equations describing the cosmic string network was $\mathrm{O}\left(a^{4}\right)$ accurate, where $a$ is the lattice spacing. This would represent a formal improvement over the standard discretization which is only $\mathrm{O}\left(a^{2}\right)$ accurate. In fact, we omitted a key part of the improved algorithm, namely, the additional plaquette combinations in the temporal direction [1], meaning that the improvement we studied was not consistently $\mathrm{O}\left(a^{4}\right)$ accurate.

Including these additional temporal plaquette contributions would lead to an implicit update for the time evolution, and so a simulation code implementing the full improvement is likely to be significantly slower than the partially improved algorithm described in the original paper.

Our methods-including the anisotropic lattice boosting-and conclusions are not otherwise affected by this observation.

[1] G. D. Moore, Nucl. Phys. B480, 689 (1996). 